\title{
No association between multiple sclerosis and the Notch3 gene responsible for cerebral autosomal dominant arteriopathy with subcortical infarcts and leukoencephalopathy (CADASIL)
}

\author{
S A Broadley, S J Sawcer, S J S Chataway, F Coraddu, A Coles, J Gray, R Roxburgh, \\ D Clayton, D A S Compston
}

\begin{abstract}
The clinical and radiological overlap between multiple sclerosis and cerebral autosomal dominant arteriopathy with subcortical infarcts and leukoencephalopathy (CADASIL; MIM 125310) raises the possibility of diagnostic confusion and suggests that pleiotropic effects of the Notch3 gene might include influencing susceptibility to multiple sclerosis. To investigate these possibilities three microsatellites markers closely flanking the Notch 3 gene in 745 simplex families with multiple sclerosis were genotyped and exon 3 and exon 4 of the gene were directly sequenced in a subset of the index members from these families $(n=93)$. No evidence for association was found in any of the three markers and none of the commoner mutations causing CADASIL were found in the sequenced patients.

(F Neurol Neurosurg Psychiatry 2001;71:97-99)
\end{abstract}

Keywords: multiple sclerosis; CADASIL; Notch3 gene

Cerebral autosomal dominant arteriopathy with subcortical infarcts and leukoencephalopathy (CADASIL; MIM 125310) is a genetic disorder caused by mutations in the Notch 3 gene located on chromosome $19 \mathrm{q} 12 .^{1}$ The disease is typified clinically by recurrent episodes of focal neurological dysfunction which eventually result in progressive spasticity, pseudobulbar palsy, and subcortical dementia. Neuroimaging with $\mathrm{T} 2$ weighted MRI shows diffuse hyperintense lesions, concentrated in periventricular white matter but also occurring in the basal ganglia and brain stem. ${ }^{2}$ These clinical features and MRI appearances together with the occasional positivity of oligoclonal bands in the CSF of affected persons, ${ }^{3}$ explain why CADASIL is sometimes confused with multiple sclerosis, particularly when presentation is atypical.

The clinical and radiological overlap between CADASIL and multiple sclerosis together with the location of the Notch3 gene in one of the most consistently supported regions (19q) of potential linkage in multiple sclerosis identified in the genome screening of the disease $\mathrm{e}^{4-7}$ suggested to us that the gene should be considered as a positional candidate. To explore this possibility we tested three microsatellite markers (D19S923, D19S1153, and D19S841) flanking the Notch3 gene ${ }^{1}$ for evidence of association, by typing them in 745 simplex multiple sclerosis families (an affected member and both parents).

A further possibility arising from the similarities between CADASIL and multiple sclerosis is that in less distinct cases the conditions might be diagnostically confused; resulting in cases of CADASIL being mistakenly included in cohorts of familial multiple sclerosis. To investigate this second possibility we screened 93 of the index cases from our simplex families with multiple sclerosis for mutations causing CADASIL. The 93 index patients screened in this analysis were selected because each had a coaffected first degree relative, and therefore more closely mimicked the autosomal dominant inheritance seen in CADASIL families.

\section{Methods}

FAMILIES

The 745 simplex families considered in this study were all recruited in the United Kingdom and were all white. The index patients from these families all satisfied Poser criteria ${ }^{8}$ for either clinically definite (89\%), laboratory supported definite $(6 \%)$, or clinically probable (5\%) multiple sclerosis, and had a mean age at onset of 26 years, a mean EDSS of 4.5 (range $0-9.5$ ) and a mean duration of disease of 13 (range 1-39) years. As expected the index patient was female in most $(75 \%)$ of the families. All three people from each family gave written consent to take part in genetic analysis and each donated a sample of venous blood from which DNA was extracted using standard methods.

GENOTYPING

All polymerase chain reactions (PCRs) were performed on Hybaid Omnigene machines using standard methods. Forward and 
reverse primer sequences were CGGCAGTAAGCCAAGATTGT and CTAGTCACTGAGTTTGGACACTTTC for D19S923, CAATGGGAGAGAAATCCTGT and CAGCCCTTCCTTTGGTTTAT for D19S1153, together with TCCTGAGCTCAGGCAAT and CCAAGCTTTGGAGATGTC for D19S841. In each case the forward primer was fluorescently labelled enabling PCR products to be analysed on Applied Biosystems 373A sequencing machines using the GENESCAN and GENOTYPER programs (Perkin Elmer). ${ }^{9}$

SEQUENCING

Joutel et $a l^{10}$ have shown that $70 \%$ of CADASIL determining mutations are found in either exon 3 or exon 4 of the Notch 3 gene and have therefore suggested that screening for CADASIL mutations should begin with the sequencing of these exons. In keeping with this recommendation we limited our screen for mutations to the sequencing of these exons, which we performed using a nested PCR approach. A fragment including both exons was amplified first (using primers CTGGGGCCATCCTGCCCTGT and AACCATCCATGGCTCCCTGC) after which a second round of PCR was used to amplify each exon alone (exon 3 was amplified using the primers TGTGCTGCCCAACCAAGCCA and ACTGACCACACCCCCGACTA, and exon 4 was amplified using the primers TAGTCGGGGGTGTGGTCAGT and CCTCTGACTCTCCTGAGTAG). Sequencing reactions were performed on MJ tetrad thermocycler machines using an FS Taq Terminator dye kit (Perkin Elmer) as in the manufacturer's instructions followed by ethanol precipitation to remove excess dye terminators. After resuspension in EDTA/formamide buffer samples were run on an ABI 373A Sequencer. Forward and reverse sequences were run for both exons allowing sequence confirmation. Sequences were analysed using sequence analysis and sequence navigator software (Perkin Elmer).

\section{STATISTICAL METHODS}

Transmission disequilibrium testing ${ }^{11}{ }^{12}$ was performed using the TRANSMIT program version $2.5 .{ }^{13}$ The program calculates empirical $\mathrm{p}$ values which fully correct for the number of tests performed within the analysis of each marker, but not across markers, using a bootstrap method.

Each marker was tested for evidence of deviation from Hardy-Weinberg equilibrium using the Arlequin program version $1.1,{ }^{14}$ this program was also used to test for evidence of linkage disequilibrium between the markers.

\section{Results}

TRANSMISSION DISEQUILIBRIUM TESTING

To test for evidence of association we typed three microsatellite markers known to map close to the Notch 3 gene in 745 simplex families with multiple sclerosis. All three of the markers are contained in a $150 \mathrm{~kb}$ fragment of chromosome $19 \mathrm{q} 12$ which also contains the
Table 1 Transmission disequilibrium testing results

\begin{tabular}{llll}
\hline Marker/haplotype & $\chi^{2}$ & $n^{*}$ & $p$ Valuet \\
\hline D19S923 & 1.94 & 3 & 0.36 \\
D19S1153 & 1.05 & 3 & 0.65 \\
D19S841 & 9.44 & 7 & 0.01 \\
$923-1153$ & 5.23 & 5 & 0.34 \\
$1153-841$ & 8.02 & 5 & 0.13 \\
$923-841$ & 4.95 & 6 & 0.56 \\
$923-1153-841$ & 1.85 & 3 & 0.62 \\
\hline
\end{tabular}

${ }^{{ } \mathrm{n}}=$ The number of alleles/haplotypes which have a frequency of greater than $5 \%$.

TThese are the empirical $\mathrm{p}$ values calculated by TRANSMIT using the boot strap method, they correct for the number of alleles/haplotypes tested.

Notch 3 gene. ${ }^{1}$ Within this fragment two of the markers map centromeric to the gene (D19S923 and D19S1188) and one telomeric (D19S841). For each marker the genotype frequencies in the index patients correspond to those expected under Hardy-Weinberg equilibrium. However, after constructing haplotypes from the family data we found modest but statistically significant linkage disequilibrium between each pair of markers.

The results of transmission disequilibrium testing are shown in table 1. Markers were tested individually, in pairs, and as a trio (in each case alleles and haplotypes with a frequency of less than 5\% were grouped together). After correcting for the number of markers and marker combinations considered, no statistically significant evidence for transmission distortion was found. Individual alleles and haplotypes (with a frequency of greater than $5 \%$ ) were also subjected to transmission disequilibrium testing. None showed statistically significant evidence of transmission distortion (data not shown).

SCREENING FOR CADASIL MUTATIONS IN MULTIPLE SCLEROSIS CASES

None of the known CADASIL causing mutations from exon 3 and exon 4 of the Notch 3 gene were found in any of the 93 patients with multiple sclerosis we sequenced, although the previously described polymorphisms from these exons were found at the expected frequencies. The synonymous G684A polymorphism in exon 4 was seen in 22 patients, all of whom were heterozygous (allele frequency=0.11). The A587G polymorphism in exon 4, which results in the replacement of histidine 170 with arginine, a previously described non-pathogenic polymorphism, was also found in one patient (allele frequency=0.005). As a positive control for our mutation screening method we also typed DNA from a patient known to have CADASIL and confirmed heterozygosity for the C499T mutation in exon 4, which causes an arginine 141 to cysteine amino acid change in the Notch 3 protein.

\section{Discussion}

Our failure to find any evidence of association between multiple sclerosis and microsatellite markers flanking the Notch3 gene (D19S923, D19S1188, and D19S841) makes it unlikely that this gene plays any significant part in determining susceptibility to the disease. Although it is impossible to exclude a very small 
effect attributable to the gene, the many families we have studied makes it unlikely that we would have missed a substantial effect. Similarly, although it is possible for indirect tests of disease association to miss important effects when the markers typed are not in transmission disequilibrium with the causative mutation, this is unlikely to account for our negative result, as the markers we have typed are individually informative and map close to the gene.

The direct sequencing of 93 patients has a $99 \%$ power to detect polymorphisms with a frequency of $5 \%$ and a $61 \%$ power to detect polymorphisms with a frequency of $1 \%$. Thus, although we cannot exclude the possibility that cases of CADASIL are mistakenly included in cohorts of familial multiple sclerosis, our failure to find any such mutations confirms that such mistakes are very uncommon. By only sequencing exon 3 and exon 4 of the Notch 3 gene we have not specifically explored the possibility that patients with CADASIL due to other mutations might be included in familial multiple sclerosis cohorts. However, as these alternate mutations account for less than 30\% of patients with CADASIL they would be expected to account for an even lower number of mistaken diagnoses than the ones from exon 3 and exon 4 (unless they are more easily mistaken for multiple sclerosis).

In typical cases the distinction between CADASIL and multiple sclerosis is easily made. However, there are practical aspects related to the management of multiple sclerosis which tend to increase the possibility of confusion in less clear cut cases. Once a patient is given the diagnosis of multiple sclerosis it is generally more likely that atypical features (such as acute phenomena) will be interpreted as unusual manifestations of their established disease, than as typical features of a rare alternative disease warranting revaluation of the diagnosis. Indeed, even if patients are reinvestigated, atypical features on testing, such as infarction on brain MRI, may be misinterpreted as coincidental cerebrovascular disease, rather than as evidence of a rare condition such as
CADASIL. Ultimately, as the diagnosis of multiple sclerosis is rarely confirmed pathologically (even after death) it is not uncommon to find that patients with CADASIL have previously been suspected of having multiple sclerosis and indeed many have carried that diagnosis for some time before the appropriate tests are performed either on themselves or on affected relatives.

We are grateful to Elizabeth Tournier-Lasserve for providing primer sequences for exons 3 and 4 of the Notch3 gene. We thank Roswell Martin and Hugh Markus for providing the positive control sample of DNA from a patient with CADASIL. We tive control sample of DNA from a patient with CADASIL. We
also thank Laura Esposito for assistance in designing primers for also thank Laura Esposito for assistance in designing primers for
the primary PCR and for supplying the nested PCR protocol. the primary PCR and for supplying the nested PCR protocol. We remain indebted to members of the ABN from throughout
the United Kingdom who referred patients. Support was from the United Kingdom who referred patients. Support was from the Multiple Sclerosis Society of Northern Ireland and Great
Britain, the Medical Research Council, and the Wellcome Trust.

1 Joutel A, Corpechot C, Ducros A, et al. Notch3 mutations in CADASIL, a hereditary adult-onset condition causing stroke and dementia. Nature. 1996;383:707-10.

2 Chabriat H, Levy C, Taillia H, et al. Patterns of MRI lesions in CADASIL. Neurology 1998;51:452-7.

3 Chabriat H, Vahedi K, Iba-Zizen MT. Clinical spectrum of CADASIL: a study of seven families. Lancet 1995;346:9349.

4 Sawcer SJ, Jones HB, Feakes R, et al. A genome screen in multiple sclerosis reveals susceptibility loci on chromosomes 6p21 and 17q22. Nat Genet 1996;13:464-8.

5 Haines JL, Ter-Minassian M, Bazyk A, et al. A complete genomic screen for multiple sclerosis underscores a role for the major histocompatibility complex. Nat Genet 1996;13: 469-71.

6 Ebers GC, Kukay K, Bulman DE, et al. A full genome search in multiple sclerosis. Nat Genet 1996;13:472-6.

7 Kuokkanen S, Gschwend M, Rioux JD, et al. Genomewide scan of multiple sclerosis in Finnish multiplex families. $\mathrm{Am}$ F Hum Genet 1997;61:1379-87.

8 Poser CM, Paty DW, Scheinberg L, et al. New diagnostic criteria for multiple sclerosis: guidelines for research protocols. Ann Neurol 1983;13:227-31.

9 Reed PW, Davies JL, Copeman JB, et al. Chromosomespecific microsatellite sets for fluorescence-based, semiautomated genome mapping. Nat Genet 1994;7:390-5.

0 Joutel A, Vahedi K, Corpechot C, et al. Strong clustering and stereotyped nature of Notch 3 mutations in CADASIL patients. Lancet 1997;350:1511-15.

11 Spielman RS, McGinnis RE, Ewens WJ. Transmission test for linkage disequilibrium: The insulin gene region and insulin-dependent diabetes mellitus (IDDM). Am 7 Hum Genet 1993;52:506-16.

12 Spielman RS, Ewens WJ. The TDT and other family-based tests for linkage disequilibrium and association. Am f Hum Genet 1996;59:983-9.

13 Clayton D, Jones H. Transmission/disequilibrium tests for extended marker haplotypes. Am f Hum Genet 1999;65: 1161-9.

14 Schneider S, Kueffer JM, Roessli D, et al, eds. Arlequin version 1.1: a software for population genetic data analysis. Switzerland: Genetics and Biometry Laboratory, University of Geneva, 1997 